\title{
Synthesis of stereoenriched piperidines via chemo-enzymatic dearomatization of activated pyridines
}

Vanessa Harawa ${ }^{1}$, Thomas W. Thorpe ${ }^{1}$, James R. Marshall ${ }^{1}$, Amelia Gilio ${ }^{2}$, Rachel S. Heath ${ }^{1}$, Antonio Angelastro ${ }^{1}$, James D. Finnigan ${ }^{3}$, Simon J. Charnock ${ }^{3}$, Gideon Grogan², Roger C. Whitehead ${ }^{1}$, Nicholas J. Turner ${ }^{1 *}$.

Affiliations

${ }^{1}$ Department of Chemistry, University of Manchester, Manchester Institute of Biotechnology, 131 Princess Street, Manchester, M1 7DN (UK).

${ }^{2}$ Department of Chemistry, University of York, Heslington, York, YO10 5DD (UK).

${ }^{3}$ Prozomix, Building 4, West End Ind. Estate, Haltwhistle, (UK).

${ }^{*}$ Corresponding author. Email: Nicholas.Turner@manchester.ac.uk

\section{Abstract}

The development of efficient and sustainable methods for the synthesis of nitrogen heterocycles is an important goal for the chemical industry. In particular, substituted chiral piperidines are prominent targets due to their prevalence in medicinally relevant compounds and their precursors. A potential biocatalytic approach to the synthesis of this privileged scaffold would be the asymmetric dearomatization of readily assembled activated pyridines. However, nature has yet to yield a suitable biocatalyst specifically for this reaction. Here, by combining chemical synthesis and biocatalysis, we present a general chemo-enzymatic approach for the asymmetric dearomatization of activated pyridines for the preparation of substituted piperidines with precise stereochemistry. The key step involves a stereoselective one-pot amine oxidase/ene imine reductase cascade to convert $\mathrm{N}$-substituted tetrahydropyridines to stereo-defined 3- and 3,4substituted piperidines. This chemo-enzymatic approach has proved useful for key transformations in the syntheses of the antipsychotic drugs Preclamol and OSU-6162, as well as for the preparation of two important intermediates in synthetic routes of the ovarian cancer monotherapeutic Niraparib.

\section{One-Sentence Summary}

A biocatalytic cascade for the asymmetric synthesis of $N$-heterocyclic therapeutic precursors including Niraparib.

Introduction

The ubiquity of saturated nitrogen heterocycles ( $N$-heterocycles) in natural products and pharmaceuticals continues to drive the development of innovative strategies for their efficient synthes is $(1,2)$. In particular, chiral piperidines are much sought after structures due to their prevalence as scaffolds in a range of bioactive molecules including market approved active pharmaceutical ingredients (APIs)(3). Nature provides highly efficient biocatalysts for the biosynthesis of $\mathrm{N}$-heterocycles $(4,5)$, offering high enantio- and regio-selectivity under benign conditions. These biocatalysts have previously enabled the development of one-pot cascade reactions to access stereo-enriched 2-, 2,6- and 2,3substituted piperidines(6-10). However, the translation of these methods to the corresponding stereoenriched 3- 
substituted and 3,4-substituted scaffolds, the core of many important therapeutic compounds (Fig. 1A), remains challenging due to difficulties in stereoselectivity control combined with limited availability of suitable starting material s.

Asymmetric chemical synthetic approaches for the preparation of 3-substituted and 3,4-disubstituted piperidines include those based on metalation/cross-coupling(11-14), Grignard Michael addition(15), ring-closure(16), and transition-metal-catalyzed dearomatization of pyridines(17-20). However, limitations are associated with all of these approaches, including high reaction temperatures, sensitivity to moisture, lack of availability of starting materials and the use of expensive non-commercial chiral ligands(11, 21). Among reported methods, the catalytic asymmetric dearomatization of pyridines is achieved by quaternization-activation of the pyridine nitrogen, permitting access to mild reduction methods to chiral piperidines (Fig. 1B, left)(17). Whilst nature has yielded pyridine synthases to prepare pyridines(22), an effective biocatalyst for their dearomatization has yet to be discovered. With this in mind, we sought to combine mild chemical reduction of pyridiniums to tetrahydropyridines (THPs) with the exquisite stereoselectivity of a biocatalytic cascade to reduce the final $\mathrm{C}=\mathrm{C}$ bond as an efficient strategy for asymmetric dearomatization of activated 3- and 3,4-substituted pyridines (Fig. 1C). Biocatalysts with broad substrate scope for the reduction of $\mathrm{C}=\mathrm{C}$ bonds require the conjugation of the alkene to an electron withdrawing group. Recently, $\mathrm{C}=\mathrm{C}$ bonds conjugated to $\mathrm{C}=\mathrm{N}$ bonds have been shown to undergo full reduction to amines through the combination of ene-reductases (EREDs) and imine reductases (IREDs)(6), as well as the newly discovered ene imine reductase (EnelREDs)(23). We reasoned that biocatalytic oxidation, using an amine oxidase (AmOx), of the THP in situ would generate the corresponding dihydropyridiniums (DHPs), generating an activated $\mathrm{C}=\mathrm{C}$ bond conjugated to the $\mathrm{C}=\mathrm{N}$ bond, which could then be reduced with these biocatalysts to generate a cascade to the desired 3- and 3,4-substituted piperidines. This cascade would complement previously reported amine oxidase AmOx-IRED deracemisation processes in which only amine oxidation and $\mathrm{C}=\mathrm{N}$ bond reduction take place $(24,25)$.

\section{Results and discussion}

A series of substituted $N$-alkyl THPs 1 b-21b was prepared in good yields (50-90\%) from activated pyridines (1a-21a) using $\mathrm{NaBH}_{4}$ as previously reported(26). Initially, we explored the conversion of THPs to piperidines using AmOxs in combination with EREDs or EnelREDs (see supplementary materials 2.1.; Figs. S1 to S5 for the complete list of THPs screened). For the first step we tested AmOx variants that have been shown to be effective biocatalysts for the oxidation of $\mathrm{N}$-alkyl $\operatorname{THPs}(27,28)$. The 6-hydroxy-D-nicotine oxidase (6-HDNO) variant, E350L/E352D(29), was found to be effective, with broad substrate scope, including oxidation of $\mathbf{1 b}$, a precursor to Preclamol. We next screened for activity for the second step, namely reduction of the $\mathrm{C}=\mathrm{C}$ bond of the $\alpha, \beta$-unsaturated iminium ion. Whereas the $p$ anel of EREDs displayed no activity, the EnelRED from an unidentified Pseudomonas sp. (EnelRED-01)(23), in combination with 6-HDNO, was effective at reducing a number of THPs and could be used to prepare piperidine $(R)$ 1c in good yield and with excellent enantioselectivity (Table S1; entry 1-3, $\geq 42 \%$ yield, 96\% ee). 
Next, we set out to identify further EneIREDs that could also generate enantioenriched 3-substituted piperidines. By screening the recently reported metagenomic IRED collection(30), in combination with the 6-HDNO variant, we were able to quickly identify biocatalysts capable of generating either enantiomer of piperidine (R/S)-1c from THP 1b (Table S2). From this screen we organized these EnelREDs into two groups: Series A (red: EnelREDs 01-04) that gave piperidine (R)-1c (Table S2 up to $>99 \%$ ee), and Series B (blue: EnelREDs 05-09) that generated the enantiocomplementary piperidine (S)-1c (Table S2, up to 96\% ee).

With effective EnelREDs for the preparation of both enantiomeric series, we probed the substrate scope of the 6HDNO-EnelRED cascade (Fig. 2). Enzymes in Series A and B accepted a variety of aryl substituents at the C-3position of the THP scaffold, affording products 1c-7c in high yields, conversion, and enantioselectivity. 5-membered heterocyclic 3-substituents such as furan 8c and thiophene 9c were also well tolerated ( $\geq 62 \%$ yield, $\geq 86 \%$ ee). Sterically demanding substrates, e.g. containing a 2-naphthyl substituent $\mathbf{1 0 b}$, were also tolerated producing $(A)$-and (B)-10c in excellent yield and stereoselectivity (73\% yield, $>99 \%$ conv., $\geq 94 \%$ ee). Additionally, a variety of $N$-alkyl substituents were accepted forming the piperidine products $1 \mathrm{c}-\mathbf{1 2 c}$ in good to excellent yields. Of these, $\mathrm{N}$-allyl $\mathbf{3 c} \mathbf{c}-\mathbf{c} \mathrm{c}$ and $N$-propargyl 12c piperidines provide useful synthetic handles that can be easily removed or further functionalized.

More hindered 3,4-disubstitituted THPs could also be reduced using the cascade, resulting in the formation of the substrate dependent cis or trans isomer. These included substrates in which both substituents were simple alkyl $(3,4-$ dimethyl) $17 \mathbf{b}$ or part of a fused bi-cyclic ring system (octahydroisoquinoline) 18b. We then probed the tolerance for a combination of alkyl and aryl substituents at C-3 and C-4. 3-Phenyl-4-methyl disubstituted compounds 19b and 20b provided the corresponding piperidines $(A)-19 c$ and $(A)-20 c$ in excellent yields and diastereoselectivity (cis major; $>96: 4 \mathrm{dr} ; \geq 87 \%$ yield). The system also tolerated the isomeric 3-methyl-4-phenyl disubstituted THP 21c. In addition to the inversion of stereochemical outcome at the C-3 position (Series A vs Series B), we also discovered some EnelREDs in Series B that provided an inverted diastereomeric configuration of the 3,4-disubstitituted piperidines 17c20c compared to Series A.

To probe the mechanism of the 6-HDNO-EnelRED cascade, the conversion of THP $6 \mathbf{b}$ to piperidine $6 \mathbf{c}$ was investigated by in situ ${ }^{19} \mathrm{~F}$ NMR reaction monitoring (Fig. 3A). After $<5$ min, formation of piperidine $6 \mathrm{c}$ was apparent and after $60 \mathrm{~min}$ the THP $\mathbf{6 b}$ was completely consumed. In the absence of the EneIRED, 6-HDNO catalyzed the previously reported aromatization of THP $\mathbf{6 b}$ to the corresponding pyridinium ion $\mathbf{6 a}$ (Fig. S6, >99\% conversion after $24 \mathrm{~h})(28)$. As expected, no direct reduction of the THP $\mathbf{6 b}$ to piperidine $6 \mathbf{c}$ was observed in the absence of $6-\mathrm{HDNO}$ suggesting that a transiently generated dihydropyridinium (DHP) is the substrate for the EneIRED reduction.

Using the cascade with THP-10b we were able to isolate the enamine 10e before full conversion to the piperidine $10 \mathrm{c}$ (see supplementary materials 4.1., 63\% yield) which strongly suggested the participation of this compound in the reaction pathway. Accordingly, enamine 10e was converted to piperidine 10c using EnelRED-01 alone, in excellent 
yield and high enantioselectivity, equivalent to the full cascade with THP-10b (Fig. 3B, top; $88 \%$ yield, 94\% ee). Deuterium labelling experiments were also implemented to further elucidate the mechanism of the EneIRED enamine reduction step. Carrying out the reduction of enamine 10e using EneIRED-01, in the presence of GDH and $D$-glucose1- $d_{1}$ to generate deuterated NAD(P)D in situ, C-2-mono-deuterated $10 \mathrm{c}-\boldsymbol{d}_{\mathbf{1}}$ was formed (80\% deuterium incorporation, $82 \%$ yield). This suggests that the enamine intermediate $10 \mathrm{e}$ may undergo protonation to the iminium before $\mathrm{NAD}(\mathrm{P}) \mathrm{H}$ hydride delivery (Fig. 3B, bottom and see supplementary materials 4.2.).

A proposed mechanism for the 6-HDNO-EnelRED cascade is outlined in Fig. 3C. For illustrative purposes, we have depicted the transformation of THP-3b to $(S)-3 c$ as this product is used in the subsequent synthesis of the key intermediate for Niraparib described below. Initially, 6-HDNO oxidation of THP-3b results in the activation of the THP $\mathrm{C}=\mathrm{C}$ bond for EnelRED-catalyzed asymmetric conjugate reduction of DHP-3d at the expense of NADPH to generate enamine $3 \mathbf{e}$ (Cycle 1; step iii). This intermediate $\mathbf{3 e}$ is expected to be in equilibrium with chiral iminium $\mathbf{3 f}$ via a nonselective protonation in solution, which has been extensively documented $(9,31,32)$. Depending on the EnelRED employed (Series A or B), the kinetic selective reduction of one enantiomer of chiral iminium $3 \mathbf{f}$, affords the enantioenriched piperidine $3 \mathrm{c}$ as the final product via reduction with a second molecule NADPH (Cycle 2; step vii). In situ epimerization of the enantiomer in $\mathbf{3 f}$ via enamine $3 \mathbf{e}$, enables a dynamic kinetic resolution (DKR) to occur to generate piperidine $(S)-3 c$ mediated by the EnelRED.

Predominantly, EnelREDs in Series A yielded (R)-piperidines whilst enzymes in Series B such as EnelRED-07 yielded the $(S)$-product. In order to gain insight into the mode of substrate binding in the active site we determined the structure of EneIRED-07 from Micromonospora sp. Rc5 to a resolution of $2.55 \AA$ in complex with NADP ${ }^{+}$using $\mathrm{X}^{- \text {ray }}$ crystallography. Crystals were obtained in the P21212 space group and featured six molecules in the asymmetric unit, representing three dimers. EneIRED-07 displays the canonical fold observed for IREDs(33, 34), with two monomers associating to form two active sites between the $\mathrm{N}$-terminal Rossmann domain of one subunit and the $\mathrm{C}$-terminal helical bundle of its neighbor (Fig. 3D).

Analysis using the DALI server(35) suggested that the IRED with the most closely related structure was the IRED from Streptosporangium roseum (PDB 5OCM) with an rmsd of $1.0 \AA$ over $288 \mathrm{C} \alpha$ atoms. Following building and refinement of the protein atoms clear omit density was observed in each active site corresponding to the cofactor NADP ${ }^{+}$. The iminium intermediate $(S)-3 f$ the preferred enantiomer for EnelRED-07 imine reduction, was modelled into the active site using Autodock Vina (Fig. 3E)(36). The model suggests that the allyl group of (S)-3f is bound within a hydrophobic pocket formed by methionine residues M125, M183 and M214 at the rear of the active site as shown; the para-bromophenyl group projects towards the front of the active site bordered by L180, W184 and the NADP ${ }^{+}$cofactor. This ligand conformation places the electrophilic carbon approximately $3.6 \AA$ from the $\mathrm{NADP}^{+}$pyridinium ring $\mathrm{C} 4$ atom, from which hydride is transferred. Modelling of the $(R)$-enantiomer of $3 \mathrm{f}$ places the allyl group at the base of the active 
site with less favorable interactions with hydrophilic residues Y225 and D238 (see supplementary materials 6.4, Fig. S13)

Finally we sought to apply the chemo-enzymatic dearomatization of activated pyridines to several target bioactive molecules (Fig. 4). First, we targeted the antipsychotic drug Preclamol. At preparative scale (1 mmol), THP-1b was converted to both $(R)-(+)-$ and (S)-(-)-preclamol 22, using EnelRED-01 and EnelRED-05 respectively. Both enantiomers were prepared in four steps from 3-(3-methoxyphenyl)pyridine and were obtained in $\geq 50 \%$ overall yield and with $96 \%$ ee (Fig. 4A and see supplementary materials 4.1.). Next, we carried out the three-step syntheses of both enantiomers of OSU6162 2c, using EneIRED-02 and EnelRED-06, and these were both accomplished in $\geq 36 \%$ overall yield and $\geq 92 \%$ ee (Fig. 4B).

To further demonstrate the application of the cascade we synthesized the two intermediates 23 and 24 en route to Niraparib (Fig. 4C), the first poly ADP ribose polymerase (PARP) inhibitor to be approved as a first-line monotherapeutic for the maintenance treatment of patients with advanced ovarian cancer(37). For route I we showed that commercially available 3-(4-bromophenyl)pyridine could be efficiently converted to piperidine (S)-3c in just three steps and $61 \%$ overall yield (99\% ee). This was followed by deallylation and $N$-Boc-protection to yield (S)-23 in $64 \%$ yield, a key intermediate in Merck's second-generation synthesis(38). Alternatively, in route II, by starting from commercially available 4-(pyridin-3-yl)aniline we converted pyridinium salt 4 a to (S)-24 in $29 \%$ overall yield and with 93\% ee, a key intermediate in Merck's first-generation synthesis(39). The general applicability of the method was also showcased by preparation of the corresponding $(R)$-enantiomers of both 23 and 24 in good yields and high enantioselectivity (see supplementary materials 4.1.).

\section{Conclusion}

In summary, we report the development of a versatile and highly efficient chemo-enzymatic dearomatization of activated pyridines for the preparation of stereo-enriched 3- and 3,4-disubstituted piperidines. The 6-HDNO-catalyzed oxidation of readily accessible THPs facilitates EneIRED-catalyzed conjugate reduction and iminium reduction to yield a broad range of chiral piperidines. The short syntheses of both enantiomers of Preclamol and OSU6162, as well as chiral precursors to Niraparib, highlight the flexibility and utility of the method presented, emphasizing the advantages of combining chemical synthesis with biocatalysis for developing new catalytic methods for the preparation of important chiral compounds. Furthermore, the increasing ability to systematically screen large panels of biocatalysts against new targets leads to the rapid identification of enzymes with applications in asymmetric synthesis.

\section{References}

1. R. D. Taylor, M. MacCoss, A. D. G. Lawson, Rings in drugs. J. Med. Chem. 57, 5845-5859 (2014).

2. M. Baumann, I. R. Baxendale, An overview of the synthetic routes to the best selling drugs containing 6 - 
membered heterocycles. Beilstein J. Org. Chem. 9, 2265-2319 (2013).

3. E. Vitaku, D. T. Smith, J. T. Njardarson, Analysis of the structural diversity, substitution patterns, and frequency of nitrogen heterocycles among U.S. FDA approved pharmaceuticals. J. Med. Chem. 57, 10257-10274 (2014).

4. D. O'Hagan, Pyrrole, pyrrolidine, pyridine, piperidine and tropane alkaloids (1998 to 1999). Nat. Prod. Rep. 17, 435-446 (2000).

5. J. A. Joule, "Natural products containing nitrogen heterocycles-some highlights 1990-2015" in Advances in Heterocyclic Chemistry (Elsevier Ltd, 2016), vol. 119, pp. 81-106.

6. T. W. Thorpe, S. P. France, S. Hussain, J. R. Marshall, W. Zawodny, J. Mangas-Sanchez, S. L. Montgomery, R. M. Howard, D. S. B. Daniels, R. Kumar, F. Parmeggiani, N. J. Turner, One-pot biocatalytic cascade reduction of cyclic enimines for the preparation of diastereomerically enriched $\mathrm{n}$-heterocycles. J. Am. Chem. Soc. 141, 19208-19213 (2019).

7. R. C. Simon, C. S. Fuchs, H. Lechner, F. Zepeck, W. Kroutil, Concise chemoenzymatic three-step total synthesis of isosolenopsin through medium engineering. European J. Org. Chem., 3397-3402 (2013).

8. R. C. Simon, F. Zepeck, W. Kroutil, Chemoenzymatic synthesis of all four diastereomers of 2,6-disubstituted piperidines through stereoselective monoamination of 1,5-diketones. Chem. Eur. J. 19, 2859-2865 (2013).

9. S. P. France, S. Hussain, A. M. Hill, L. J. Hepworth, R. M. Howard, K. R. Mulholland, S. L. Flitsch, N. J. Turner, One-pot cascade synthesis of mono-and disubstituted piperidines and pyrrolidines using Carboxylic Acid Reductase (CAR), $\omega$-Transaminase ( $\omega-T A)$, and Imine Reductase (IRED) biocatalysts. ACS Catal. 6, 37533759 (2016).

10. L. J. Hepworth, S. P. France, S. Hussain, P. Both, N. J. Turner, S. L. Flitsch, Enzyme cascades in whole cells for the synthesis of chiral cyclic amines. ACS Catal. 7, 2920-2925 (2017).

11. P. Schäfer, T. Palacin, M. Sidera, S. P. Fletcher, Asymmetric Suzuki-Miyaura coupling of heterocycles via Rhodium-catalysed allylic arylation of racemates. Nat. Commun. 8 (2017).

12. T. Jia, P. Cao, B. Wang, Y. Lou, X. Yin, M. Wang, J. Liao, A Cu/Pd cooperative catalysis for enantioselective allylboration of alkenes. J. Am. Chem. Soc. 137, 13760-13763 (2015).

13. Y. Zhou, C. Liu, L. Wang, L. Han, S. Hou, Q. Bian, J. Zhong, A concise enantioselective synthesis of (S)preclamol via asymmetric catalytic Negishi cross-coupling reaction. Synlett. 30, 860-862 (2019).

14. D. Antermite, D. P. Affron, J. A. Bull, Regio-and stereoselective palladium-catalyzed C(sp3)-H arylation of pyrrolidines and piperidines with C(3) directing groups. Org. Lett. 20, 3948-3952 (2018). 
15. D. Chamorro-Arenas, A. A. Nolasco-Hernández, L. Fuentes, L. Quintero, F. Sartillo-Piscil, Transition-metal-free multiple functionalization of piperidines to 4-substituted and 3,4-disubstituted 2-piperidinones. Chem. Eur. J. 26, 4671-4676 (2020).

16. L. Zhou, D. W. Tay, J. Chen, G. Y. C. Leung, Y. Y. Yeung, Enantioselective synthesis of 2-substituted and 3substituted piperidines through a bromoaminocyclization process. Chem. Commun. 49, 4412-4414 (2013).

17. M. Renom-Carrasco, P. Gajewski, L. Pignataro, J. G. de Vries, U. Piarulli, C. Gennari, L. Lefort, Asymmetric hydrogenation of 3-substituted pyridinium salts. Chem. Eur. J. 22, 9528-9532 (2016).

18. S. O. Thorberg, L. Gawell, I. Csöregh, J. L. G. Nilsson, Large scale synthesis and absolute configuration of (-)3-ppp, a selective dopamine autoreceptor agonist. Tetrahedron. 41, 129-139 (1985).

19. W. Li, H. Yang, R. Li, H. Lv, X. Zhang, Kinetic resolution of racemic 3,4-disubstituted 1,4,5,6-tetrahydropyridine and 3,4-disubstituted 1,4- dihydropyridines via Rh-catalyzed asymmetric hydrogenation. ACS Catal. 10, 26032608 (2020).

20. K. Kubota, Y. Watanabe, K. Hayama, H. Ito, Enantioselective synthesis of chiral piperidines via the stepwise dearomatization/borylation of pyridines. J. Am. Chem. Soc. 138, 4338-4341 (2016).

21. C. A. Busacca, D. R. Fandrick, J. J. Song, C. H. Senanayake, "Transition metal catalysis in the pharmaceutical industry" in Applications of Transition Metal Catalysis in Drug Discovery and Development (John Wiley \& Sons, Inc., 2012), pp. 1-24.

22. J. W. Bogart, A. A. Bowers, Thiopeptide Pyridine Synthase TbtD catalyzes an intermolecular formal aza-DielsAlder reaction. J. Am. Chem. Soc. 141, 1842-1846 (2019).

23. N. J. Turner, T. W. Thorpe, J. R. Marshall, V. Harawa, R. E. Ruscoe, A. Cuetos, J. D. Finnigan, A. Angelastro, F. Parmeggiani, S. J. Charnock, R. M. Howard, R. Kumar, D. S. B. Daniels, G. Grogan, Discovery of a multifunctional biocatalyst for asymmetric conjugate reduction-reductive amination. Submitted (2021), doi:10.21203/rs.3.rs-640129/v1.

24. R. S. Heath, M. Pontini, S. Hussain, N. J. Turner, Combined Imine Reductase and Amine Oxidase catalyzed deracemization of nitrogen heterocycles. ChemCatChem. 8, 117-120 (2015).

25. S. Ju, M. Qian, J. Li, G. Xu, L. Yang, J. Wu, A biocatalytic redox cascade approach for one-pot deracemization of carboxyl-substituted tetrahydroisoquinolines by stereoinversion. Green Chem. 21, 5579-5585 (2019).

26. J. E. Baldwin, L. Bischoff, T. D. W. Claridge, F. A. Heupel, D. R. Spring, R. C. Whitehead, An approach to the manzamine alkaloids modelled on a biogenetic theory. Tetrahedron. 53, 2271-2290 (1997). 
27. A. S. Kalgutkar, N. Castagnoli, Synthesis of novel MPTP analogs as potential monoamine oxidase B (MAO-B) inhibitors. J. Med. Chem. 35, 4165-4174 (1992).

28. A. Toscani, C. Risi, G. W. Black, N. L. Brown, A. Shaaban, N. J. Turner, D. Castagnolo, Monoamine Oxidase (MAO-N) whole cell biocatalyzed aromatization of 1,2,5,6-tetrahydropyridines into pyridines. ACS Catal. 8, 8781-8787 (2018).

29. R. S. Heath, M. Pontini, B. Bechi, N. J. Turner, Development of an R-selective amine oxidase with broad substrate specificity and high enantioselectivity. ChemCatChem. 6, 996-1002 (2014).

30. J. R. Marshall, P. Yao, S. L. Montgomery, J. D. Finnigan, T. W. Thorpe, R. B. Palmer, J. Mangas-Sanchez, R. A. M. Duncan, R. S. Heath, K. M. Graham, D. J. Cook, S. J. Charnock, N. J. Turner, Screening and characterization of a diverse panel of metagenomic imine reductases for biocatalytic reductive amination. Nat. Chem. 13, 140-148 (2021).

31. Y. Hai, M. Chen, A. Huang, Y. Tang, Biosynthesis of Mycotoxin Fusaric Acid and application of a PLPdependent enzyme for chemoenzymatic synthesis of substituted L-pipecolic acids. J. Am. Chem. Soc. 142, 19668-19677 (2020).

32. M.-W. Chen, X.-F. Cai, Z.-P. Chen, L. Shi, Y.-G. Zhou, Facile construction of three contiguous stereogenic centers via dynamic kinetic resolution in asymmetric transfer hydrogenation of quinolines. Chem. Commun. 50, $12526-12529$ (2014).

33. M. Rodríguez-Mata, A. Frank, E. Wells, F. Leipold, N. J. Turner, S. Hart, J. P. Turkenburg, G. Grogan, Structure and activity of NADPH-dependent reductase Q1EQE0 from Streptomyces kanamyceticus, which catalyses the R-selective reduction of an imine substrate. ChemBioChem. 14, 1372-1379 (2013).

34. G. A. Aleku, S. P. France, H. Man, J. Mangas-Sanchez, S. L. Montgomery, M. Sharma, F. Leipold, S. Hussain, G. Grogan, N. J. Turner, A reductive aminase from Aspergillus oryzae. Nat. Chem. 9, 961-969 (2017).

35. L. Holm, Benchmarking fold detection by DaliLite v.5. Bioinformatics. 35, 5326-5327 (2019).

36. O. Trott, A. J. Olson, AutoDock Vina: improving the speed and accuracy of docking with a new scoring function, efficient optimization, and multithreading. J. Comput. Chem. 23, 455-461 (2009).

37. C. J. Lord, A. Ashworth, PARP inhibitors: Synthetic lethality in the clinic. Science. 355, 1152-1158 (2017).

38. C. K. Chung, P. G. Bulger, B. Kosjek, K. M. Belyk, N. Rivera, M. E. Scott, G. R. Humphrey, J. Limanto, D. C. Bachert, K. M. Emerson, Process development of C-N cross-coupling and enantioselective biocatalytic reactions for the asymmetric synthesis of Niraparib. Org. Process Res. Dev. 18, 215-227 (2014). 
Foley, P. Fernandez, W. Hu, S. P. Keen, P. Mullens, D. Muzzio, P. Sajonz, L. Tan, R. D. Wilson, G. Zhou, G. Zhou, Development of a fit-for-purpose large-scale synthesis of an oral PARP inhibitor. Org. Process Res. Dev. 15, 831-840 (2011).

Acknowledgments: We thank Dr Sam Hart and Dr Johan P. Turkenburg for assistance with X-ray data collection and the Diamond Light Source for access to beamline 103 under proposal number mx-24948 and Dr Lucian Pirvu for assistance in generating the figures.

Funding: V.H. acknowledges a DTP award from the UK Biotechnology and Biological Sciences Research Council (BBSRC). T.W.T. is supported by a CASE award from the BBSRC and Pfizer (BB/M011208/1). J.R.M. is supported by a CASE award from the UK Industrial Biotechnology Innovation Centre (IBioIC) and BBSRC. A.G. is supported by a CASE award from Pfizer. R.S.H. is supported by the European Research Council (ERC) (Grant no. 742987) and the UK Engineering and Physical Sciences Research Council (EPSRC), BBSRC and AstraZeneca (EP/S005226/1). A.A. is supported by the EPSRC, BBSRC and AstraZeneca (EP/S005226/1). N.J.T. is grateful to the ERC for the award of an Advanced Grant (Grant no. 742987).

Author contributions: N.J.T., R.C.W., G.G. devised and supervised the project. V.H. and A.A. performed mechanistic studies. V.H. and T.W.T carried out substrate scope reactions. A.G. and G.G. obtained X-ray quality crystals and solved the crystal structures. A.G., V.H. and G.G. performed crystallographic and docking studies. V.H. carried out preparative scale reactions. V.H. and T.W.T synthesized substrates and standards. J.R.M., J.D.F. and S.J.C. performed the genetic identification, cloning and bioinformatics. V.H., J.R.M. and R.S.H produced and purified the biocatalyst. N.J.T., R.C.W., G.G., S.J.C., J.D.F., A.A., R.S.H., A.G., J.R.M., T.W.T. and V.H. wrote the manuscript and generated the figures.

Competing interests: The authors declare no conflicts of interest.

Data and materials availability: All data are available in the main text or the supplementary materials. Crystallographic structure of EneIRED-07-NADP ${ }^{+}$has been deposited in the Protein Databank (PDB) with accession code 7 OSN.

\section{SUPPLEMENTARY MATERIALS}

Materials and Methods

Supplementary Text

Figs. S1 to S59 
References (40-51)

A Representative Bioactive Piperidines<smiles>Fc1ccc(C2CCNCC2COc2ccc3c(c2)OCO3)cc1</smiles><smiles>C=CC(=O)N1CCCC(n2nc(-c3ccc(Oc4ccccc4)cc3)c3c(N)ncnc32)C1</smiles>

Ibrutunib<smiles>NC(=O)c1cccc2cn(-c3ccc(C4=CCCNC4)cc3)nc12</smiles>

Niraparib
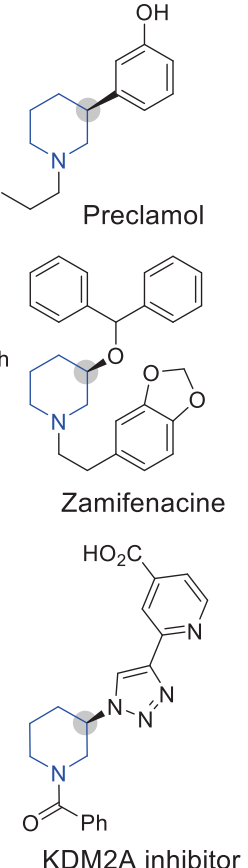

Zamifenacine
B

Previous Work:

Metal-catalysed dearomatisation

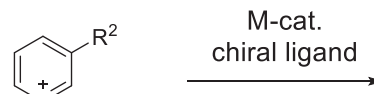

$\mathrm{R}^{1} \mathrm{X}^{-}$ chiral ligand

1

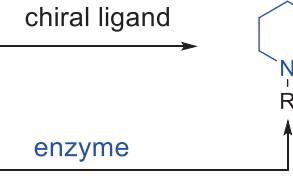

unknown

high-value products

pyridinium salt prepared from abundant pyridine

dynamic kinetic transformations

C

This Work:

Chemo-enzymatic dearomatisation approach of pyridines
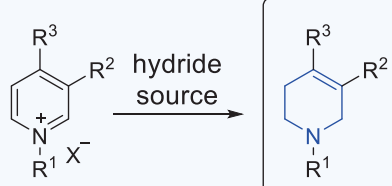

AmOx
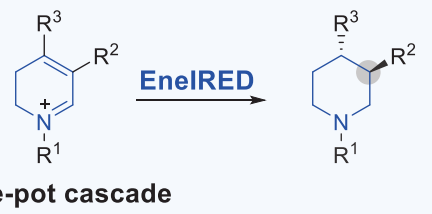

Figure 1: High value stereo-enriched 3- and 3,4-substituted piperidines and strategies for their synthesis. (A) Representative examples of biologically active chiral substituted piperidines. (B) Previous Work: Asymmetric transition metal-catalyzed synthesis of 3-substituted piperidines. (C) This Work: Chemo-enzymatic dearomatization of pyridines for the synthesis of chiral 3- and 3,4-substituted piperidines. 


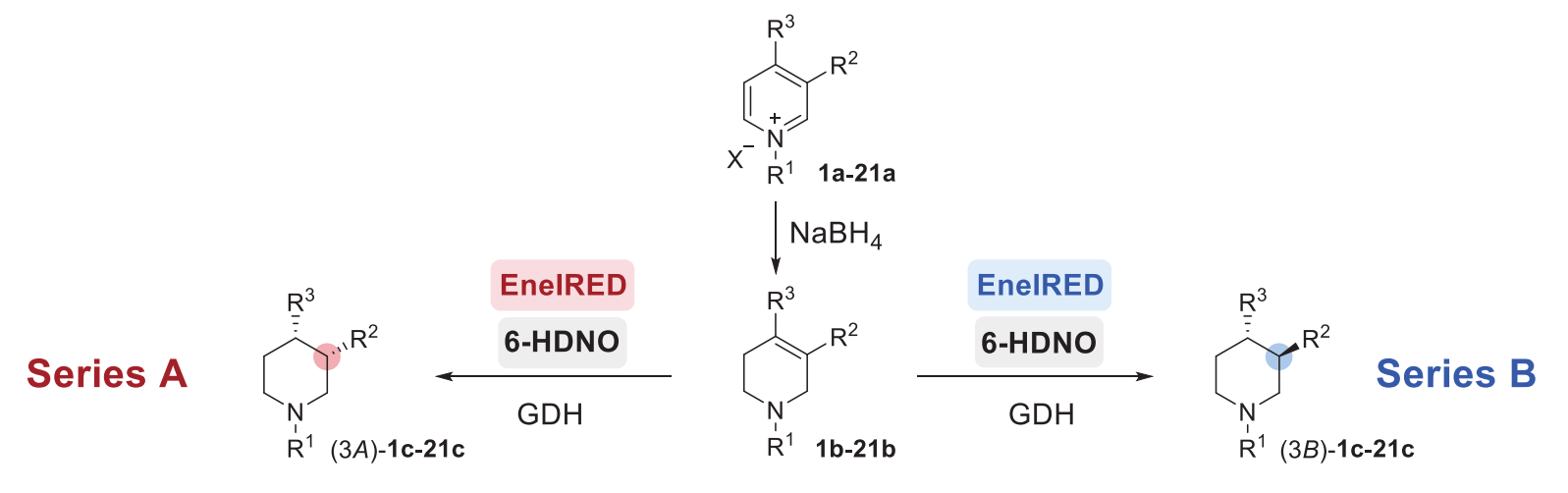

3- or 4-substituted

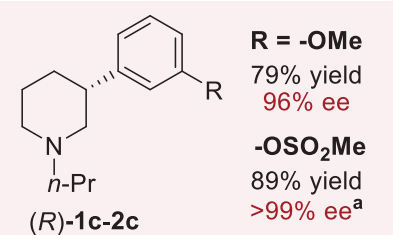

$(R)-1 \mathrm{c}-2 \mathrm{c}$<smiles>[R]c1ccc([C@H]2CCCN(CC)C2)cc1</smiles>

(A)-5c-7c

-OMe

$78 \%$ yield

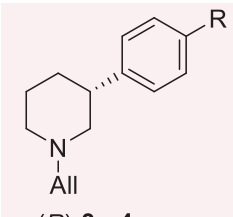

$(R)-3 \mathrm{c}-4 \mathrm{c}$

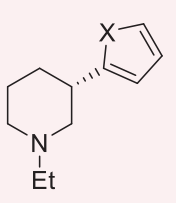

(A)-8c-9c $>99 \%$ ee

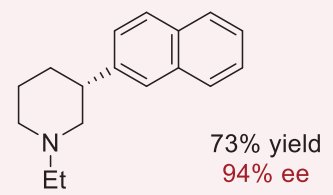

(A)-10c<smiles>[R]N1CCC[C](C)C1</smiles>

(A)-13c-14c
$\mathbf{R}=-\mathbf{E t}$ $42 \%$ yield

$-B n$ $49 \%$ conv

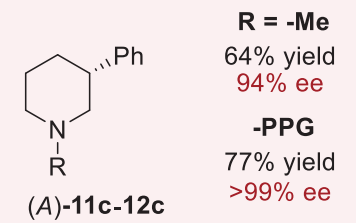<smiles>[R]C1CCN(CC)CC1</smiles>

15c-16c $64 \%$ yield -PPG $>99 \%$ ee

$\mathbf{R}=-\mathbf{M e}$ $34 \%$ yield

-Ph

$62 \%$ yield<smiles>CC(C)N1CCCC(c2cccc(F)c2)C1</smiles>

(S)-1c-2c<smiles>[R]c1ccc(C2CCCN(CC)C2)cc1</smiles>

(B) $-5 \mathrm{c}-7 \mathrm{c}$ $85 \%$ yield $86 \%$ ee

$\mathbf{R}=-\mathbf{M e}$

(B)-10c

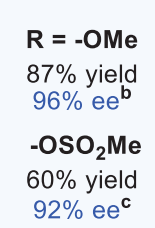

$$
\mathbf{R}=\mathbf{- H}
$$

$\mathbf{R}=-\mathbf{H}$ $93 \% \mathrm{ee}^{\mathrm{c}}$

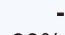

$>99 \%$ conv. $>99 \%$ ee $^{\mathrm{d}}$ $-\mathrm{OMe}$ $>99 \%$ conv. $99 \% \mathrm{ee}^{\mathrm{d}}$

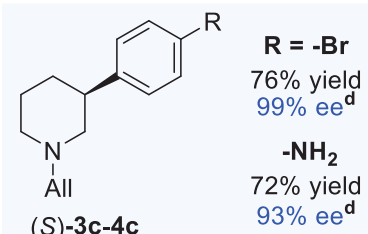

(S)-3c-4c<smiles></smiles>

(B)-8c-9c

$\mathbf{R}=-\mathbf{O}$ $>99 \%$ conv. $98 \% \mathrm{ee}^{\mathrm{c}}$

-S $>99 \%$ conv. $>99 \% \mathrm{ee}^{\mathrm{c}}$

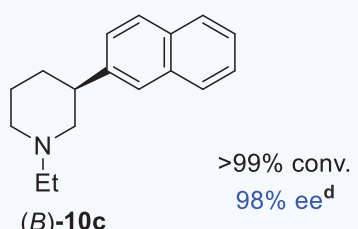<smiles>[R]C1CCN(CC)CC1</smiles>

$\mathbf{R}=\mathbf{- M e}$ $>99 \%$ conv. $^{d}$

-Ph $>99 \%$ conv., $^{\text {d }}$ $>99 \%$ conv.
$28 \% \mathrm{ee}^{\mathbf{b}}$

\section{3,4-disubstituted}

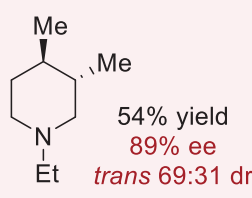

(A)-17c

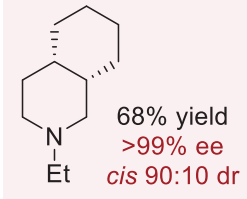

(A)-18c

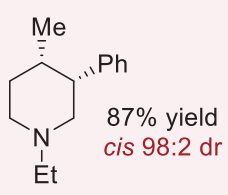

(A)-19c

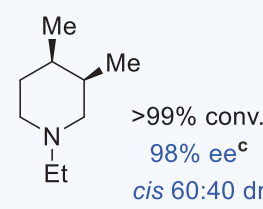

(B)-17c

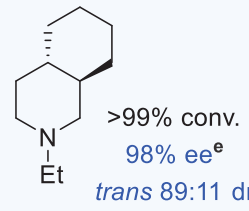

(B)-18c

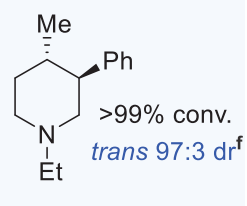

(B)-19c

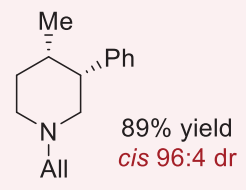

(A)-20c

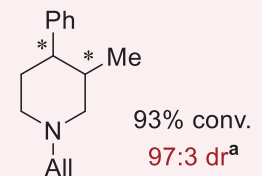

(A)-21c

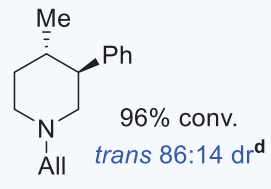

(B)-20c

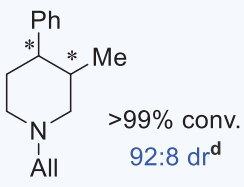

(B)-21c

Figure 2: Scope of chemo-enzymatic dearomatization of activated pyridines. Series A and Series B provide $(R)$ and (S)-configuration respectively at C-3. Reaction conditions (preparative scale): purified 6-HDNO (1 mg/mL), 
EneIRED CFE (10 mg/mL), THP (1 mmol), glucose (5 mmol), GDH CDX-901 $(0.5 \mathrm{mg} / \mathrm{mL}), \mathrm{NADP}^{+}(0.5 \mathrm{mmol})$, DMSO $(1 \% \mathrm{v} / \mathrm{v}), 100 \mathrm{mM} \mathrm{KPi} \mathrm{pH} \mathrm{7.0,16-24} \mathrm{h,} 30{ }^{\circ} \mathrm{C}, 200 \mathrm{rpm}$ (analytical conditions): purified 6-HDNO (0.5 mg/mL), EneIRED CFE ( $4 \mathrm{mg} / \mathrm{mL}), 10 \mathrm{mM}$ THP $(5 \mu \mathrm{mol}), 50 \mathrm{mM}$ glucose $(25 \mu \mathrm{mol})$, GDH CDX-901 $(0.1 \mathrm{mg} / \mathrm{mL}), 0.5 \mathrm{mM}$ $\left.\mathrm{NADP}^{+}(0.05 \mu \mathrm{M}), 2-16 \mathrm{~h}\right)$. All examples use EneIRED-01 except; aEnelRED-02, 'beneIRED-05, 'EneIRED-06, ${ }^{d}$ EnelRED-07, eEneIRED-08 and ${ }^{\mathrm{f} E n e l R E D-09 . ~ S e e ~ s u p p l e m e n t a r y ~ m a t e r i a l s ~ f o r ~ m o r e ~ d e t a i l s . ~}$ 
A

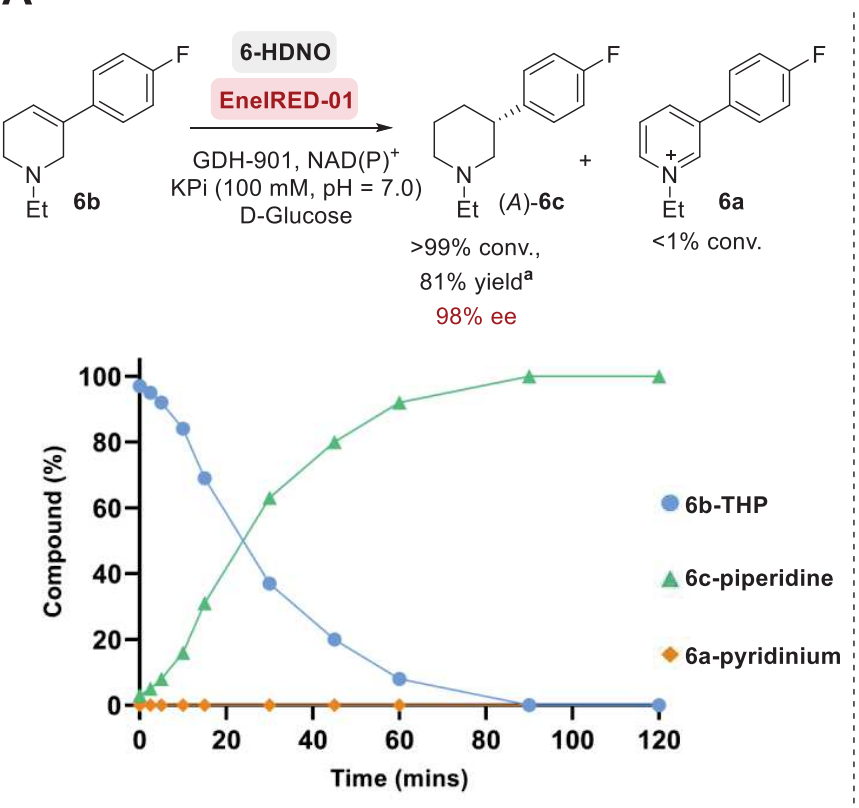

B

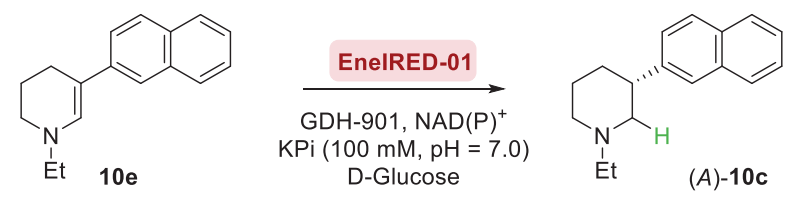

$88 \%$ yield $94 \%$ ee

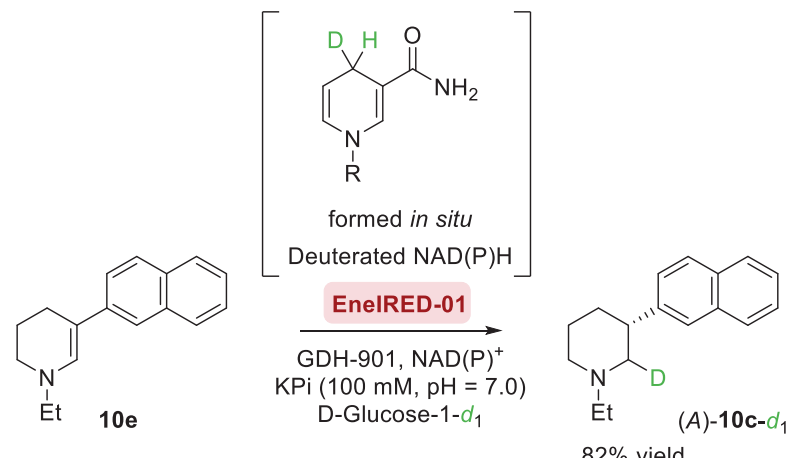

$80 \%$ deuterium incorporation
C

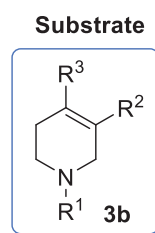

$\mathrm{R}^{1}=$ allyl

$\mathrm{R}^{2}=p-\mathrm{Br}-\mathrm{Ph}$

$\mathrm{R}^{3}=\mathrm{H}$

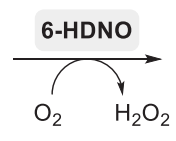

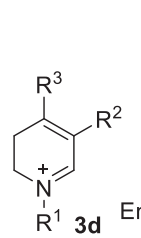

EnelRED: NAD(P)H/ 3d<smiles>CC1CCCCC1C</smiles>

Conjugate reduction

$\mathrm{R}^{1}$ 3d EnelRED: NAD $(\mathrm{P}) \mathrm{H}$ cycle 1

in

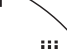

Enamine

intermediate in solution

diate in solution

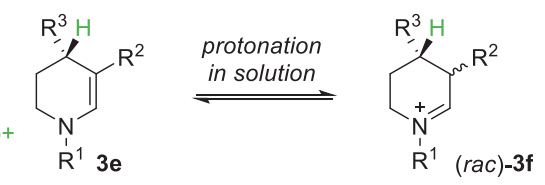

$\begin{array}{ll}1 & \\ \mathrm{R}^{1} & \mathbf{3 e}\end{array}$ $\mathrm{R}^{1}$ (rac)-3f
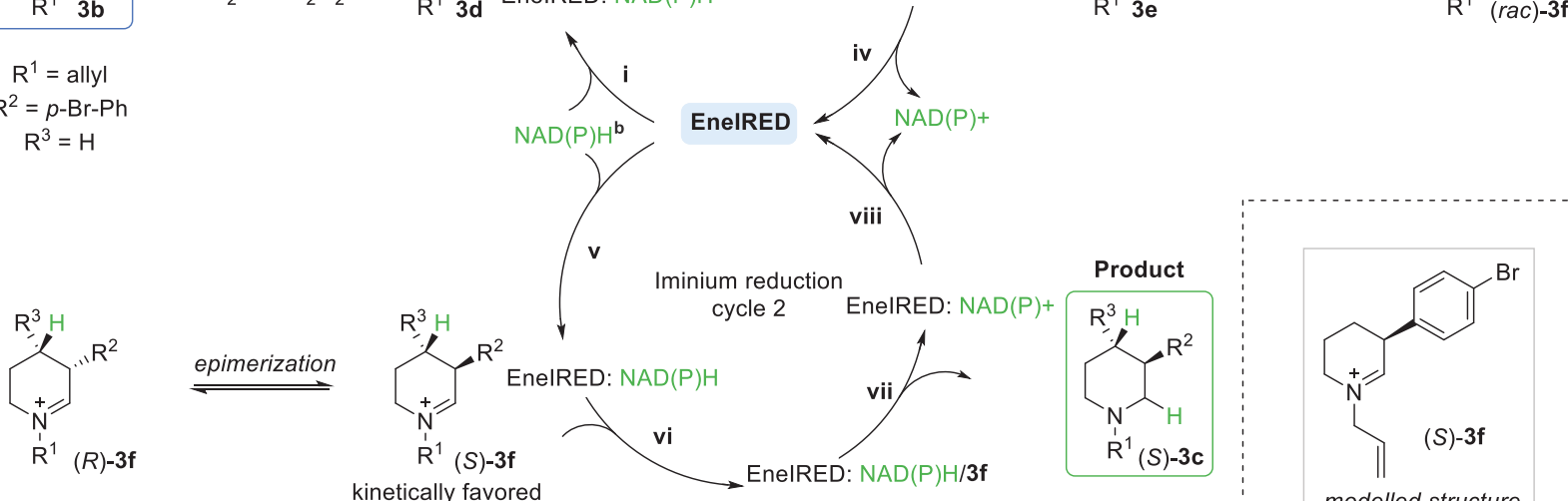
kinetically favored

\section{D}

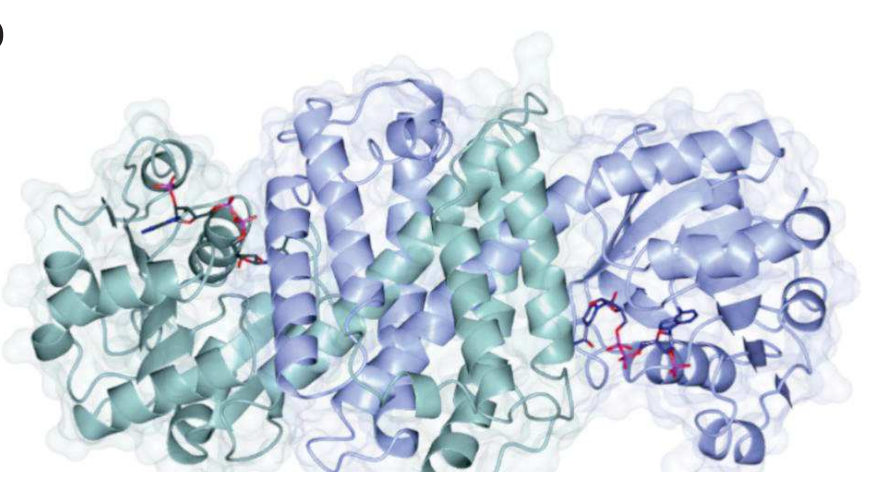

E

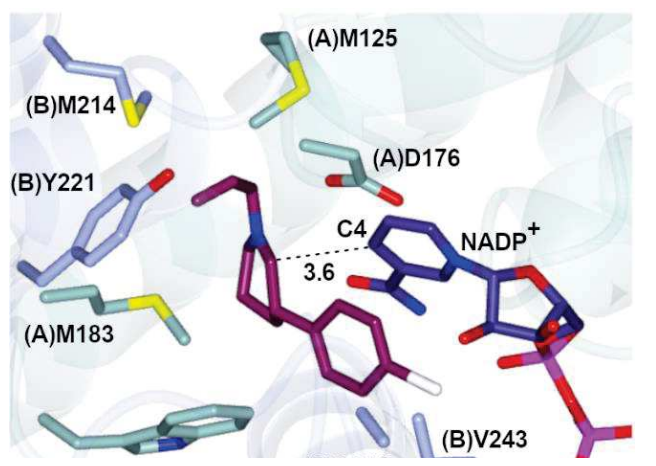

Figure 3: Mechanistic studies and proposed mechanism for the 6-HDNO-EneIRED cascade. (A) Kinetic profile

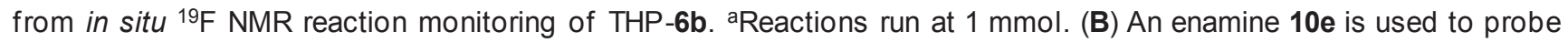
the role of this species as an intermediate in the cascade. (C) Proposed catalytic sequence for the AmOxEnelRED 
biocatalytic cascade. bTwo equivalents of $\mathrm{NAD}(\mathrm{P}) \mathrm{H}$ consumed to form piperidine (D) Structure of the dimer of EnelRED-07 in ribbon format with subunits shown in green and blue. NADP ${ }^{+}$can be seen bound at the two active sites. (E) Active site of EneIRED-07 with the (S)-enantiomer of iminium ion intermediate $3 \mathbf{f}$ modelled into the active site. NADP ${ }^{+}$and (S)-3f are shown in cylinder format with carbon atoms in grey and purple respectively. The electrophilic carbon of the intermediate is $3.6 \AA$ from the $\mathrm{NADP}^{+}$pyridinium ring $\mathrm{C}-4$ atom, from which hydride is transferred.

A Synthesis of (-)-Preclamol<smiles></smiles>

(i) $\mathrm{NaBH}_{4}$

(ii) 6-HDNO EnelRED-05

(iii) aq. $48 \% \mathrm{HBr}$
$67 \%$ yield ( 3 steps)

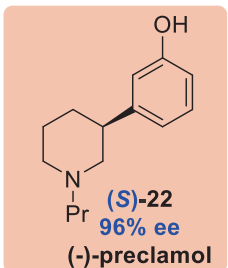

B

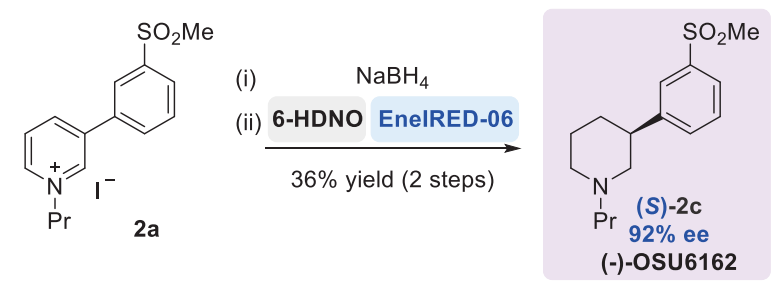

C<smiles>C=CC[n+]1cccc(-c2ccc(Br)cc2)c1</smiles>

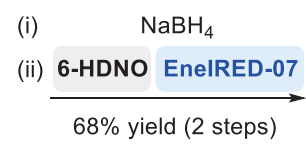

Two chemo-enzymatic approaches to Niraparib

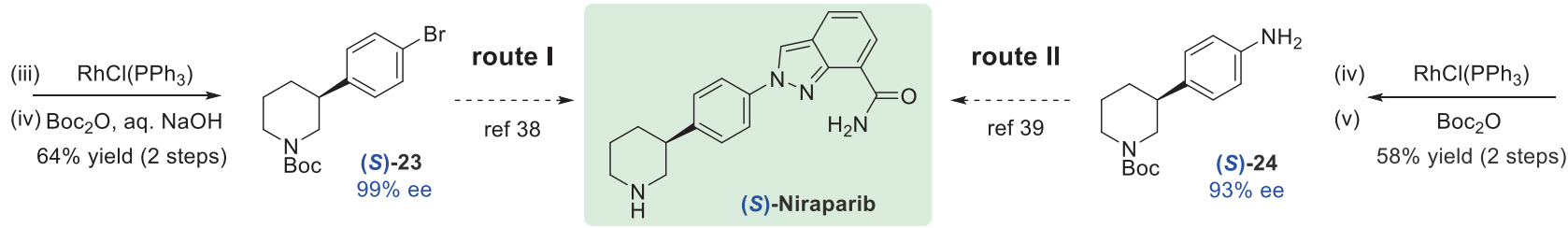

Figure 4: Application of the chemo-enzymatic dearomatization of pyridines for the preparation of APls. (A)

Synthesis of the anti-psychotic drug (-)-preclamol. (B) Synthesis of (-)-OSU6162. (C) Two synthetic routes to Niraparib. 